\title{
Establishment of Surface Topography Simulation Model with Considering Vibration and Wear of Ball-end Milling
}

\author{
XiuLin Sui ${ }^{1}$, Yi Zheng ${ }^{2}$, JinGang Jiang and XinMin Feng \\ College of Mechanical and Power Engineering, Harbin University of Science and \\ Technology \\ Harbin Heilongjiang 150080, China \\ ${ }^{1}$ suixiulin@sina.com, ${ }^{2} 953832836 @ q q . c o m$
}

\begin{abstract}
Focusing on the influence of various factors of cutter and work-piece in milling process, such as force, wear and vibration, using discrete method to compute the trajectory of each points, and get the coordinates values of discrete points on the cutting edge/at any time. A new method has been proposed to obtain the dynamic response of discrete points by transforming deformation of those values into work-piece coordinate system. At the same time, a new policy of retention has been proposed to-avoid inadequate statistics and large number of statistics. Retaining coordinates which can effectively influence the formation of surface topography, the prediction model of surface topography can be established with the consideration of force, wear and dynamic response on the ball-end mill. This prediction model of surface topography has beengroved to be effective and practicable by an experiment.
\end{abstract}

Keywords: ball-end cutter; surface topography force; wear; vibration

\section{Introduction}

When using a ball-end cutter for milling, various factors may cause deviation between theoretical trajectory and actual trajectory. Comprehensive consideration is the key for the success of the simulation of the surface topography [1].

There are two existing methods of ball-end milling surface topography simulation. One is the use of the path enyelope surface of cutter to intersect with the work-piece. Lmani BM [2] has given a surface topography model of intersection between envelope surface and workpiece. CHIOU [3] proposed maximum potential field path planning, with good computational efficiency. Fangyu Peng [4] proposed a bounding box to obtain the surface topography. The other is Z-map method. OMAR [5] studied the influence of different axis rotation error in the milling cutte system for the surface contour. Some points have influence on the surface topography, but, these methods are only equivalent to random census part of the point, and cannot accurately describe the points. And those surface topography simulation methods rarely involves in force, vibration and wear. A double-buffering mechanism [6] involved force and vibration, but it does not include wear.

In this paper, a surface topography simulation model has been presented, which contains the force, vibration, wear information. In this model, coordinate file of useful points can be updated with the machining, it also avoid the inadequate statistical and large number of statistical. Accuracy of the model has been proven by experiments 


\section{Establishment of Trajectory Equations of Cutting Edge Discrete Points}

\subsection{Point on the Cutting Edge Mathematical Description}

Ball-end cutter is the main tool of milling, an arbitrary point $Q$ on the cutting edge, relative to the center of the sphere, can be represented by $(u, v, w$,$) . As follows:$

$$
\left[\begin{array}{c}
u \\
v \\
w
\end{array}\right]=\left[\begin{array}{c}
R \sin a \cos (\tan \beta \ln [\cot (a / 2)]+\theta) \\
R \sin a \sin (\tan \beta \ln [\cot (a / 2)]+\theta) \\
-R \cos a
\end{array}\right]
$$

$\beta$ is the helix angle of ball-end mill; $z$ is the number of cutting edges; $R$ is the radius of the ball-end mill. If you select a time interval $t$, then $\theta$ is the angle of rotation of the cutting edge during the time.

\subsection{Trajectory of Discrete Points on the Cutting Edge}

Point coordinates of sphere center need to be converted into work-piece coordinate system. Translation vector can be computed by the following formula.

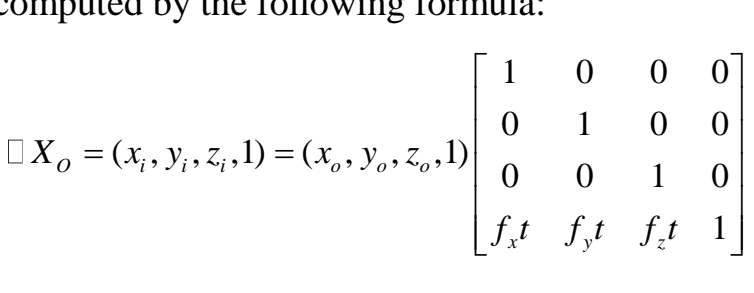

Let $M_{1}=\left[\begin{array}{cccc}1 & 0 & 0 & 0 \\ 0 & 1 & 0 & 0 \\ 0 & 0 & 1 & 0 \\ f_{x} t & f_{y} t & f_{z} t & 1\end{array}\right], \quad x_{o}, y_{o}, z$ is the center Coordinates of the sphere when $t_{i}=0, f_{x}, f_{y}, f_{z}$ is the feed amount of the cutter in the $\mathrm{x}, \mathrm{y}, \mathrm{Z}$ direction, $x_{i}, y_{i}, z_{i}$ is the center coordinates of the sphere when $t_{i}=t$.

Because pre- and post-inclination angle $\beta_{f}, \beta_{n}$ and number of cutting edges $z$, the trajectory of the center of the ball alsoneed to convert. Transformational matrix of the pre-inclination post-inclination angle and number of cutting edges is $M_{2} M_{3} M_{4}$.

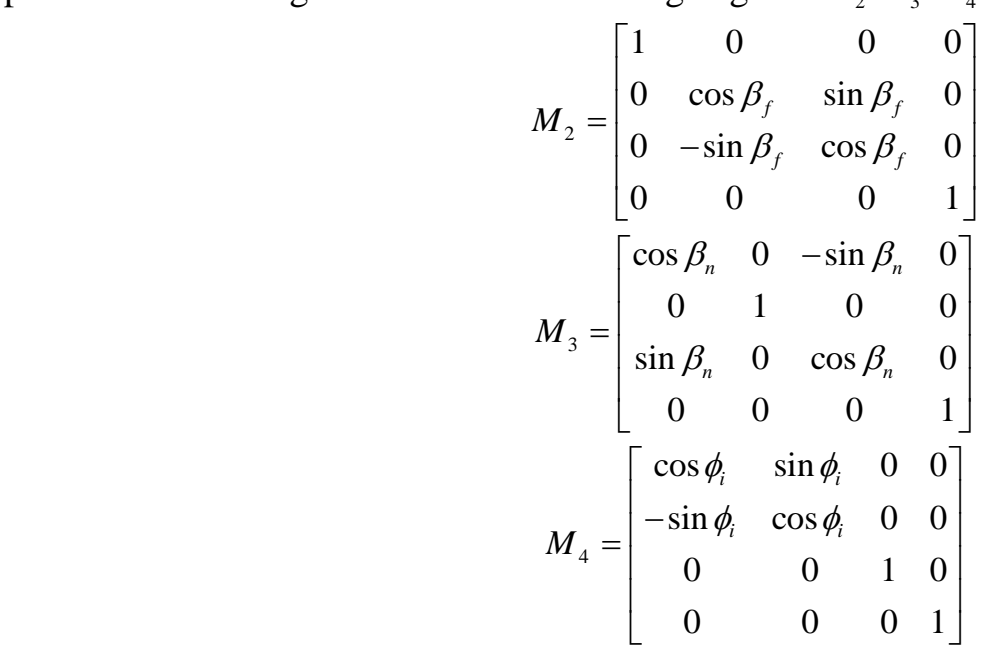


Fully consider formula (1) - (5), the following formula can be obtained.

$$
X_{T P}=\left(x_{i}, y_{i}, z_{i}, 1\right)=\left(x_{0}, y_{0}, z_{0}, 1\right) M_{1}+(u, v, w, 1) M_{2} M_{3} M_{4}
$$

$X_{T P}$ is the coordinate vector of any point at any time, and the coordinate $P_{i}\left(x_{i}, y_{i}, z_{i}\right)$ of any point in the cutting edges can be obtained at any time. Integrated over time, trajectory of points can be obtained in the work-piece coordinate system.

\section{Modification of the Trajectory}

\subsection{Modification of the Trajectory based on Force of Cutter}

The cutter is assumed to be a cantilever beam. $O_{1} X_{1} Y_{1} Z_{1}$ is established as the moying coordinate system. $O_{I}$ is the origin point of coordinate system $O_{1} X_{1} Y_{1} Z_{1}$, it is also the center of sphere.

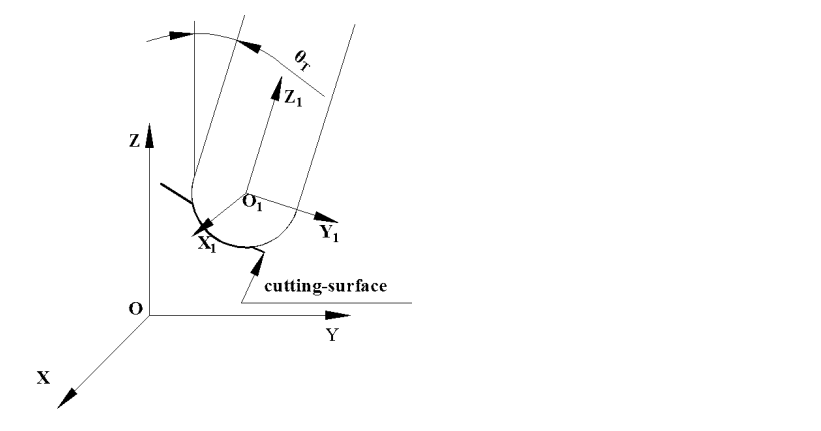

\section{Figure 2. Coordinate System Conversion}

Regardless of the force, point of the theoretic center to be the origin of coordinate, the direction of axis for the $z$ axis. The deformation of $\mathrm{X}$ and $\mathrm{Y}$ direction at the point $(0,0,-z)$, $\chi_{x}(\theta, z)$ and $\chi_{y}(\theta, z)$ l7 can be expressed as:

$$
\left\{\begin{array}{l}
\chi_{x}(\theta, Z)=\frac{F_{y}(\theta)}{2 E R^{4}}\left[\left\langle Z_{F X}-Z\right\rangle^{3}-(L-Z)^{3}+3(L-Z)^{2}\left(L-Z_{F x}\right)\right] \\
\chi_{0}\left(Q Z=\frac{F_{y}(\theta)}{2 E R^{4}}\left[\left\langle Z_{F y}-Z\right\rangle^{3}-(L-Z)^{3}+3(L-Z)^{2}\left(L-Z_{F y}\right)\right]\right.
\end{array}\right.
$$

$E$ is the elastic modulus of milling, $R$ is the radius of cutter, $F_{y}(\theta)$ and $F_{x}(\theta)$ are the cutting forces of the $x$ and $y$ direction when cutter rotated $\theta$ degree, $L$ is the length of the cutter, $Z$ is the position of the cutter that generates tool deflection; $Z_{F x}$ and $Z_{F y}$ are the distance between cutting force point and the origin of the coordinate along the axis of $\mathrm{x}$ and $\mathrm{y}$ directions. Function of \langle\rangle is a window function.

$$
\langle a\rangle=\left\{\begin{array}{l}
a, a \geq 0 \\
0, a<0
\end{array}\right.
$$

The deformation can be converted into the work-piece coordinate system by the following formula. 


$$
M_{5}=\left[\begin{array}{cccc}
1 & 0 & 0 & 0 \\
0 & 1 & 0 & 0 \\
0 & 0 & 1 & 0 \\
\chi_{x}(\theta, 2) & \chi_{y}(\theta, 2) & 0 & 1
\end{array}\right]
$$

\subsection{Modification of the Trajectory based on Vibration of Cutter}

The cutting point be the origin point, the direction of cutter axis be the z-axis, the normal vector of the intersection in $Z_{1} O_{1} Y_{1}$ plane, established following coordinate system $O_{2} X_{2} Y_{2} Z_{2}$. The relationship between two coordinate systems, $O_{1} X_{1} Y_{1} Z_{1} O_{2} X_{2} Y_{2} Z_{2}$, can be shown as Fig.3.

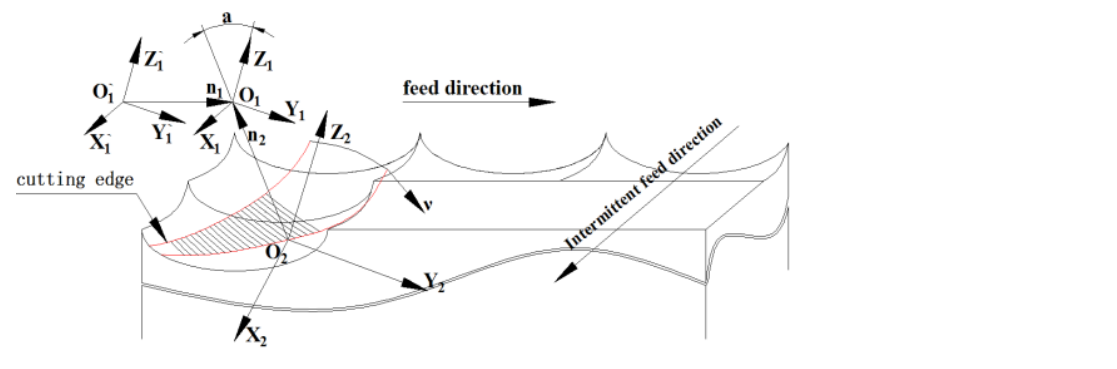

Figure 3. The Relationship of Transformation between the Coordinate System

Vibration function of the ball-end mill 8 can be obtained, as the follows:

$$
Y_{r}(z)=\operatorname{ch} \hat{\beta}_{r} z-\cos \beta_{r} z+\varepsilon_{r}\left(\operatorname{sh} \beta_{r} z+\sin \beta_{r} z\right)
$$

This equation corresponds to the natural frequency of the milling

$$
\omega_{0}=\beta^{2} \sqrt{\frac{E J}{\rho A}}(r=1,2,3 \cdots)
$$

Parameters $\beta_{r}$ an be determined by the flexural vibration characteristic equation of milling cutter $\cos \beta \operatorname{ch} \beta L=C, E$ is the elastic modulus of milling cutter. The time of sampling about the point on the cutting edge, $t$ is set to $m / \omega_{r}\left(m>200 \omega_{r}\right)$. Transformation matrix can be obtained as follows.

$$
\left(u_{i}, v_{i}, w_{i},\right)=\vec{n}_{2}+\left(0, Y_{r}, z,\right)\left[\begin{array}{ccc}
\cos \sigma & \sin \sigma & 0 \\
-\sin \sigma & \cos \sigma & 0 \\
0 & 0 & 1
\end{array}\right]
$$

\subsection{Modification of the Trajectory based on Wear of Cutter}

In the following coordinate system $O_{1} X_{1} Y_{1} Z_{1}$, the wear [9] along XYZ directions can be expressed as the follows:

$$
\left\{\begin{array}{c}
M_{d x}=\Delta A_{h}(\Delta t) \sin \psi(i, a, z) \\
M_{d y}=\Delta A_{h}(\Delta t) \cos \psi(i, a, z) \\
M_{d z}=\Delta A_{z}(\Delta t)
\end{array}\right.
$$

$M_{d x} 、 M_{d y}$ is necessary to compensate respectively for the amount of radial direction and $\mathrm{y}$ 
direction along the $\mathrm{X}$ decomposition rate of compensation, $M_{d z}$ is the compensation required by the axial. The transformation matrix is as follows:

$$
M_{6}=\left[\begin{array}{cccc}
1 & 0 & 0 & 0 \\
0 & 1 & 0 & 0 \\
0 & 0 & 1 & 0 \\
-M_{d x} & -M_{d y} & -M_{d z} & 1
\end{array}\right]
$$

\subsection{Discrete Point Trajectory Equation}

Fully consider formula (6) - (15), any point on the cutting edge, coordinates $\left(x_{i}, y_{i}, z_{i}\right)$ in the work-piece coordinate system, can be computed by the following formula.

$$
\left(x_{i}, y_{i}, z_{i}, 1\right)=\left(x_{0} y_{0} z_{0} 1\right) M_{1} M_{5} M_{6}+\left(u_{i}, v_{i}, w_{i}, 1\right) M_{2} M_{3} M_{4}
$$

\section{Surface Topography Simulation and Experiment}

\subsection{Discrete Point Trail Reservation}

Not all points on the cutting edge were involved in the cutting, and in order to avoid unnecessary coordinate statistics, participants of the cutting ed ge need to be determined.

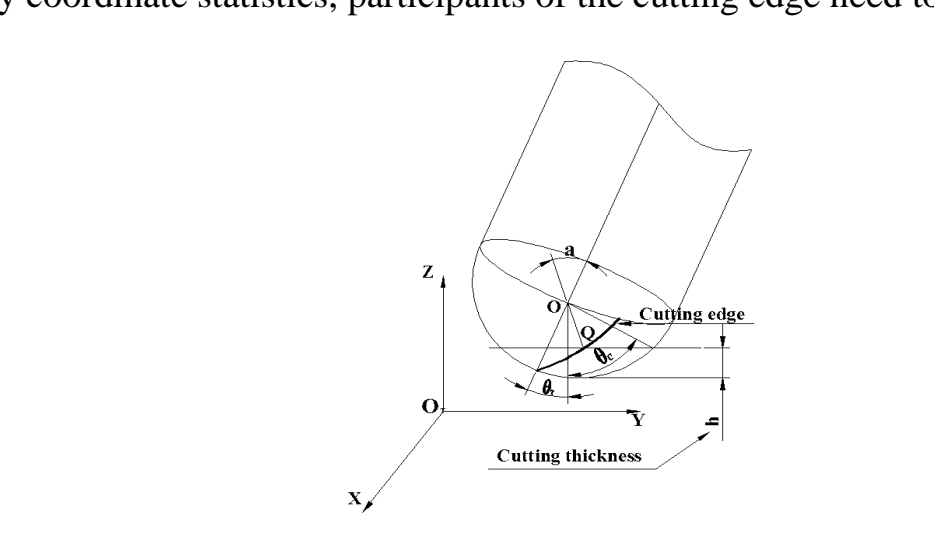

\section{Figure 5. Participants of the Cutting Edge}

Shown in Figure 5, $\theta_{\top}$ is the angle between the rotating axes and the normal of the deepest point of the cutting. By calculation, the policy of reservation of trajectory can be obtained as follows:

If $a>\theta_{\mathrm{r}}+\theta_{\mathrm{C}}$, the point is never involved in cutting.

If $a \leq \theta_{\top}$, the point has been involved in cutting, information need to be reserve.

If $\theta_{\mathrm{T}}<a<\theta_{\mathrm{T}}+\theta_{\mathrm{C}}$, the point is intermittent participation. If the following equation holds, information of trajectory needs to be reserve. $\theta_{0}=(n-1) \frac{2 \pi}{z},(0 \leq n \leq z), \quad \theta$ is the angle of rotation of the cutting edge during the time interval $t$.

$$
\cos \left(\theta_{0}+\theta\right)<\frac{3-2 \cos \theta_{\mathrm{C}}}{\sin a \sin \theta_{\mathrm{T}}}-\frac{\tan a}{\tan \theta_{\mathrm{T}}}
$$


Otherwise According to the third case. Set $t$ to be the time intervals, coordinates for each discrete point can be gotten at the time. Coordinates of points formed at different times to meet the following type $\left(t_{m}>t_{n+1}\right)$, as Figure 6 .

$$
\left\{\begin{array}{l}
x_{n}<x_{m}<x_{n+1} \\
y_{n}<y_{m}<y_{n+1}
\end{array}\right.
$$

\section{Figure 6. The Method of Retention for Discrete Points}

When the other point fall into this area, the distance of this point and $Q\left(x_{m}, y_{m}, z_{m}\right)$ from the design surface e need to be compared, then the minimum alue also need to be remained.

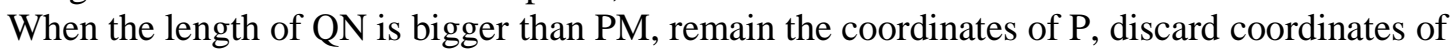
Q.

\subsection{Logic of Surface Topography Sinulation}

MATLAB and visual $\mathrm{C}++$ mixed progrâmming is used in Software, MATLAB is used to generate $\mathrm{m}$ files; VISUAL $\mathrm{C}++$ is used to generate the main control program, and call MATLAB in a variety of procedures. The logic of surface topography simulation can be shown as Figure 7.

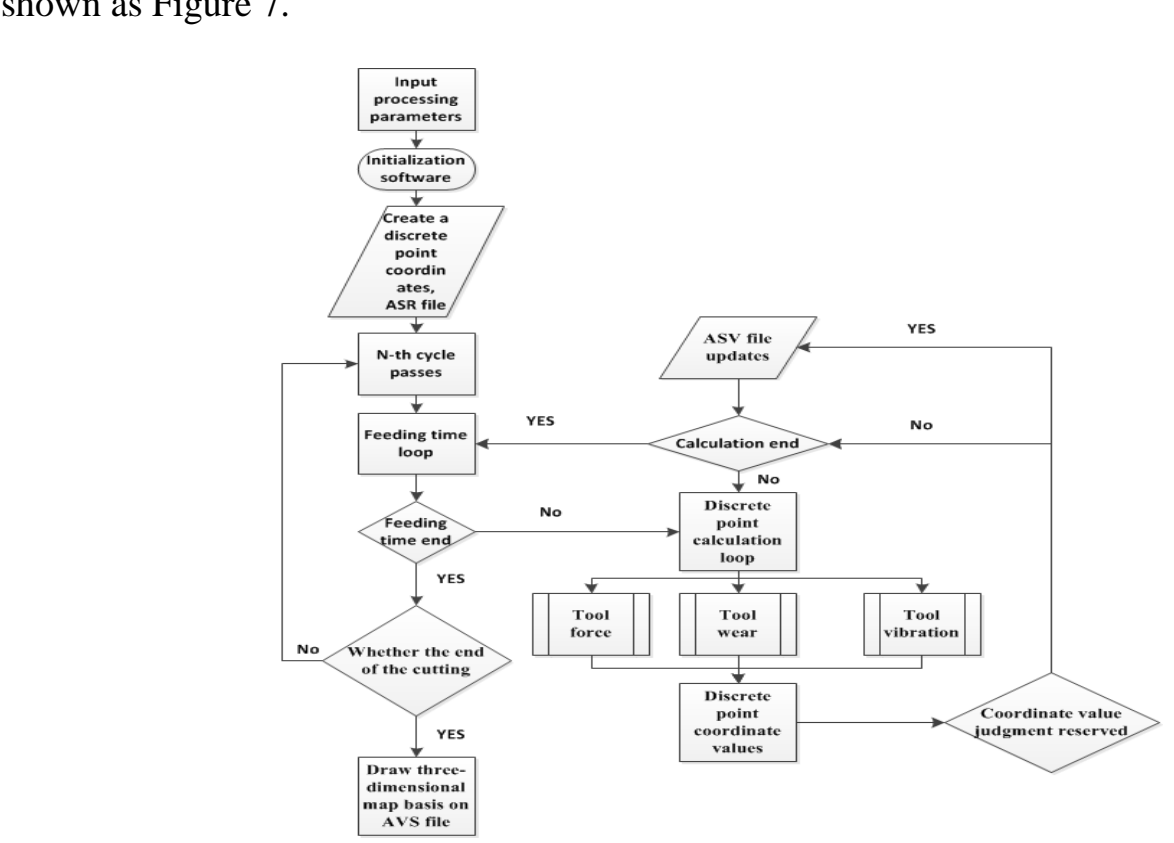

Figure 7. System Logic Diagram 


\subsection{Milling Experiment}

In order to find out the accuracy of the model, a series of experiments need to be carried out. Three - dimensional graphics of Helmet and experimental processing is shown as Figure 8.
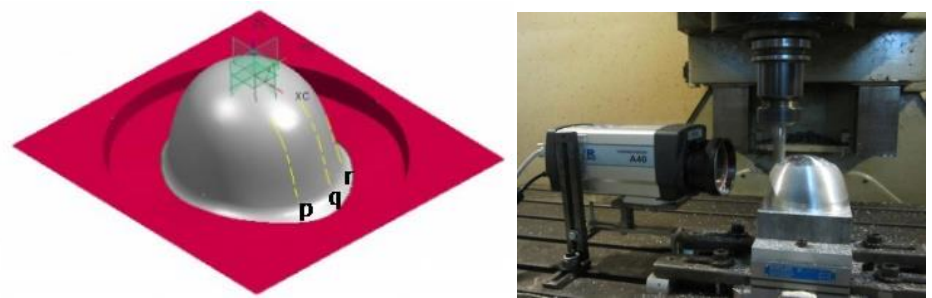

Figure 8. Helmet 3D Model and Processing of Experimental

The condition of experimental shown as follow.

Table 1. Experimental Conditions

\begin{tabular}{|c|c|}
\hline Experimental parameters & Numerical \\
\hline \multicolumn{2}{|l|}{ Spindle speed (r/min) } \\
\hline Tool Feed $(\mathrm{mm} / \mathrm{min})$ & $\mathrm{mmm} / \mathrm{min}, 1500 \mathrm{~mm} / \mathrm{min}$ \\
\hline Cutting depth (mm) & \\
\hline Tool material & 1 \\
\hline Tool diameter (mn & 10 \\
\hline Cutter teeth & 2 \\
\hline & Ball End \\
\hline Tool helix a & $30^{\circ}$ \\
\hline Work-piece material & AISI 2024 \\
\hline $\mathrm{CNC}$ machining equipm & XH715 Machining Center \\
\hline & $\begin{array}{c}\text { Coordinate Measuring Machine } \\
\text { Reference } 600\end{array}$ \\
\hline the maximu & $(1.0+\mathrm{L} / 350)$ \\
\hline
\end{tabular}

\subsection{Analysis of Experimental and Simulation Result}

Within the greenzone of each line to take five test points on the three lines $p, q, r$. Simulation value and the actual value comparison processing in Table 2. Figure 9 shows the comparison of actual microscopic surface and microscopic surface of simulation.

\section{Cable 2. Error Comparison between Experiment and Simulation}

\begin{tabular}{cccc}
\hline Measuring & Measuring points & Simulation value $(\mathrm{mm})$ & Error \\
\hline \multirow{2}{*}{ line } & $(46.8401,-50.2002,-0.0379)$ & $(46.8401,-50.2002,-0.0292)$ & 0.0087 \\
& $(46.8339,-50.3118,-1.0325)$ & $(46.8339,-50.3118,-1.0407)$ & -0.0082 \\
$\mathrm{p}$ & $(46.6695,-50.2045,-2.0291)$ & $(46.6695,-50.2045,-2.0443)$ & -0.0152 \\
& $(46.8446,-50.2002,-3.0397)$ & $(46.8446,-50.2002,-3.0299)$ & 0.0098 \\
& $(46.7164,-50.6863,-4.0395)$ & $(46.7164,-50.6863,-4.0245)$ & 0.0150 \\
& Z average value-2.03114 & Z average value-2.04272 & Average error \\
\hline $\mathrm{q}$ & $(46.0445,-52.4472,-0.0981)$ & $(46.0445,-52.4472,-0.1093)$ & -0.01138 \\
\hline
\end{tabular}




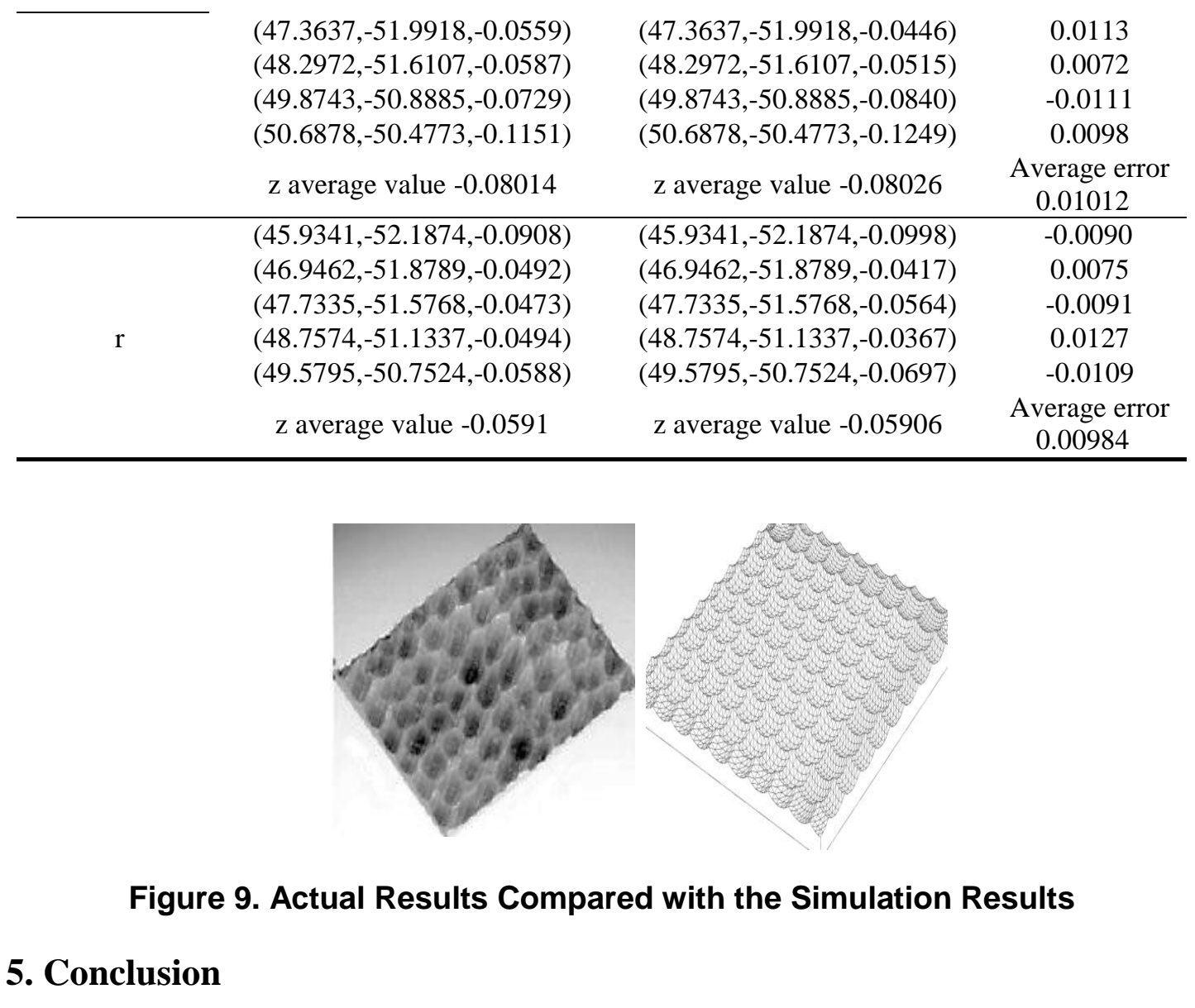

(1) The establishment of multiple coordinate systems is the key for the success of ball-end milling simulation model. All of the kariables are converted into the work-piece coordinate system, which make the prediction model of surface topography can be established with the consideration of force, wear and dynamic response. Through experimental verification model is correct.

(2) Not all of the poins on the cutting edge are involved in machining. Some of them are intermittent cutting. So 3 new policy of retention has been proposed to avoid inadequate statistics and large number of statistics.

(3) The results of experiment show that the values of simulation model and test values have a good agreement. But this model also has errors, if the heat of tool and work-piece, and thermal coupling power into account, the accuracy of the simulation model would have further improvement.

\section{Acknowledgment}

This research was sponsored by Excellent Academic Leaders Project of Harbin science and technology innovation talents of special fund (2013RFXXJ064). 


\section{References}

[1] W. Fang, "Study on topography simulation of high speed NC machined surfaces", Jinan Shandong University, (2007).

[2] B. M. Lmani and M. A. Elbestawi, "Geometric simulation of ball-end milling operations", Journal of manufacturing Science and Engineering, vol. 123, (2001), pp. 177-184.

[3] C. J. Chiou and Y. S. Lee, "A machining potential field approach to tool path generation for multi-axis sculptured surface machining”, Computer Aided Design, vol. 34, no. 5, (2002), pp. 357-371.

[4] P. Fangyu and Fangzhenglong, "Tree-dimensional surface topography simulation of ultra-precision milling based on point cloud", Journal of Huazhong University of Science and technology (Natural science Edition), vol. 40 , no. 8 , (2012).

[5] O. Omar, T. E. Wardany and M. A. Elbestawi, "An improved cutting force and surface topography prediction model in end milling", International Journal of Machine Tools and Manufacturing, vol. 47, no. 78, (2007), pp. 1263-1275.

[6] X. Liang and Z. Yao, "Dynamic-based Simulation for Machined Surface Topography in 5-axis Ball-end Milling", Chinese Journal of Mechanical Engineering, vol. 48, no. 1, (2013), pp. 185-192.

[7] X. Sui, P. Zhao, C. Zhang, P. Zhang and N. Hu, "Modeling and compensation analysis of ball-end milling cutter wear", Proceedings of 2011 International Conference on Electronic and Mechanical Engineering and Information Technology, EMEIT, vol. 6, (2011), pp. 3293-3296.

[8] X. Sui, P. Zhao and Y. Wang, "Research for NC milling error analysis and compensation", Applied Mechanics and Materials, vol. 121-126, (2012), pp. 2167-2173.

[9] S. Xiulin, Z. Chunhong, Z. Ping, H. Na and Z. Ping, "Research on finite element simulation processing method for flatness prediction", 2011 International Conference on Mechanical Engineering, vol. 148-149, (2011), pp. 88-92.

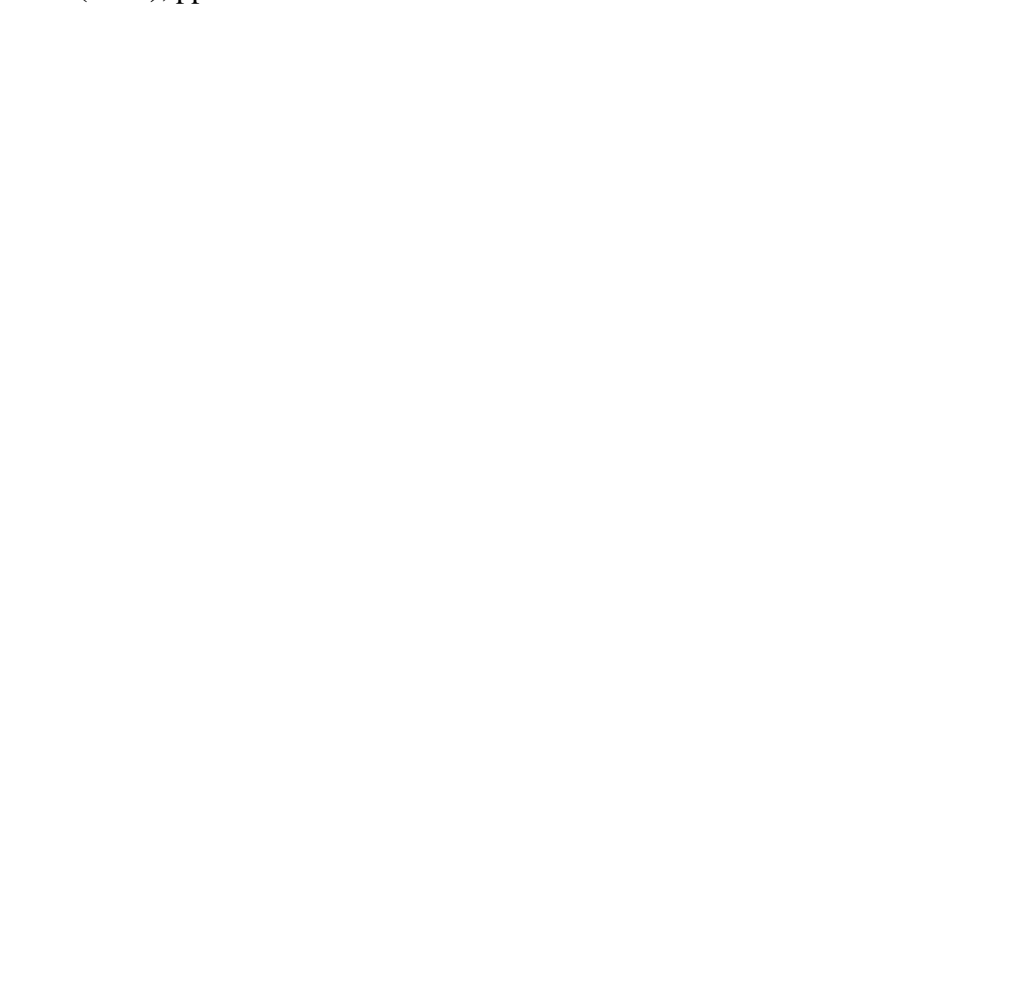


International Journal of Smart Home

Vol.8, No.1 (2014)

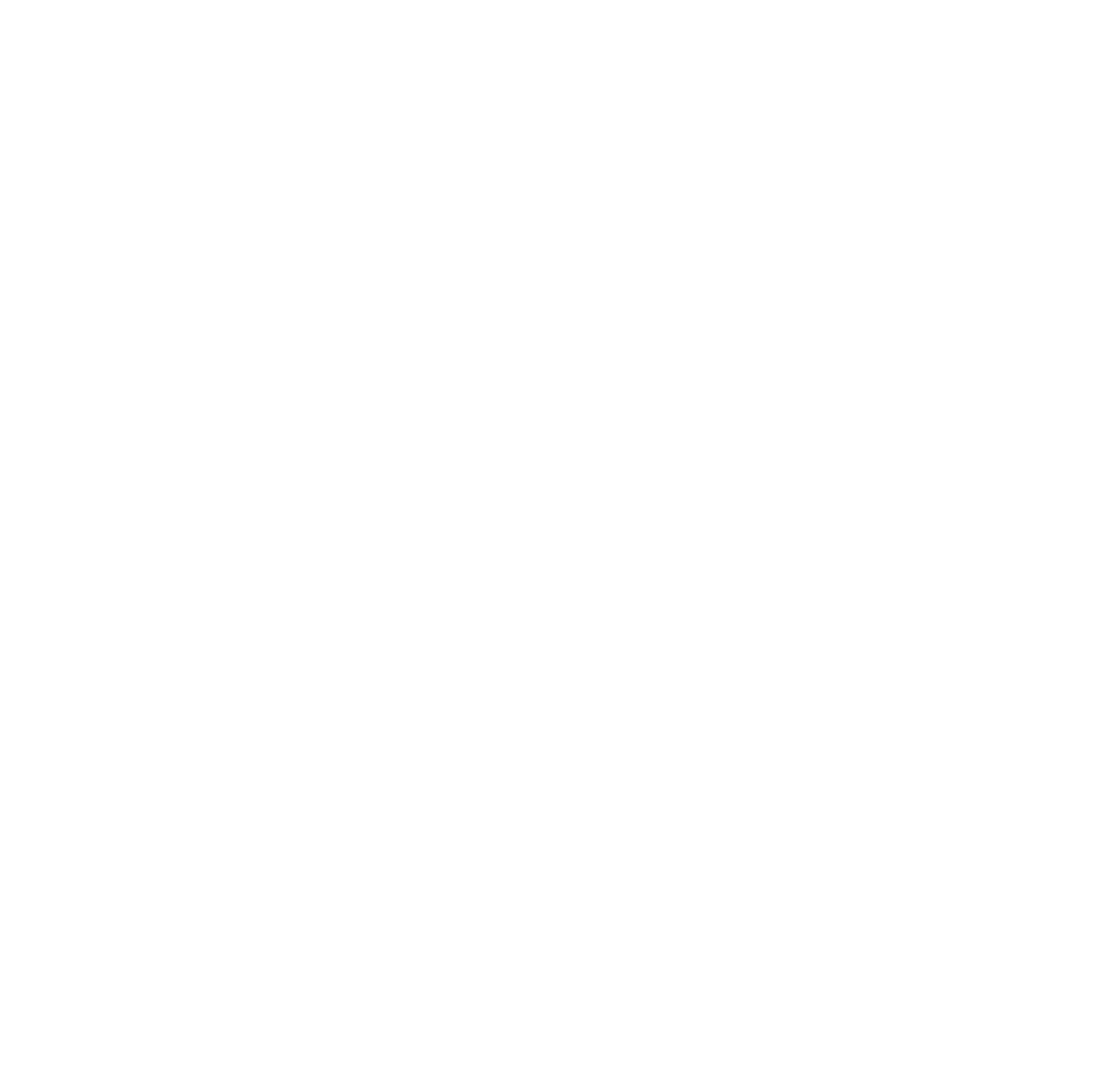

